enciencias e

Artículo/Article
Sección/Section B

\section{Sugerencias para la toma de datos \\ en eventos de biología reproductiva de aves}

\section{Héctor Cadena-Ortiz}

Aves Quito (Club de Observadores de Aves), Ecuador.

Museo Ecuatoriano de Ciencias Naturales del Instituto Nacional de Biodiversidad, Calle

Rumipamba 341 y Av. de los Shyris. Casilla 17-07-8976, Quito, Ecuador.

Autor para correspondencia/Corresponding author, e-mail:fercho cada@yahoo.es

\section{Suggestions for data collection in bird biology reproductive events}

\section{Abstract}

There is a major need to collect and publish information on the natural history of Ecuadorian birds. The vast majority of articles in this field are published in English. There remains a need to standardize the terms and types of data collected in the field. Here, I detail the considerations that should be taken when observing a reproductive event to avoid overlooking any data, and making more complete comparisons. I also present different terms and their translations used in articles on this topic

Keywords: Breeding biology, measures, terminology, types of nest, weights.

\section{Resumen}

Persiste la necesidad de generar y publicar información sobre la historia natural de las aves ecuatorianas. La mayoría de los artículos en este campo se publican en inglés. Surge la necesidad de estandarizar términos y datos tomados en campo. En el presente trabajo, detallo las consideraciones que se deberían tomar al momento de observar un evento reproductivo, para no pasar por alto ningún dato y poder realizar comparaciones más completas. También presento diferentes términos en inglés y en español usados en artículos sobre este tema.

Palabras clave: Biología reproductiva, medidas, pesos, terminología, tipos de nido.
Si bien el número de estudios en biología reproductiva de aves en el Neotrópico ha aumentado, aún contrasta con el conocimiento más completo disponible en otras regiones, como Europa [1,2]. En el Neotrópico aún falta por describirse nidos de muchas especies o identificar si las descripciones realizadas son el patrón o la excepción. Además, se requiere comprender los parámetros de reproducción, evaluar si hay o no temporalidad determinar la puesta usual de cada especie y cómo esto se refleja en general en la dinámica de las poblaciones [3]. En las últimas décadas, el incremento del conocimiento de la biología reproductiva de las aves del Neotrópico, y en particular del Ecuador, ha tenido sustento en varias revistas regionales y globales, siendo las principales: Ornitología Neotropical y Cotinga [4]. Es así que entre 2014 y 2015, se publicaron 10 artículos sobre biología reproductiva de aves de Ecuador entre ambas revistas, todos en idioma inglés.

El Ecuador es un país megadiverso y un atributo importante de esta diversidad son las aves, representadas en el país por 1608 especies confirmadas y documentadas [5]. Sin embargo, para la mayoría de estas especies aún hay poca información sobre su biología reproductiva: comportamiento reproductivo, ritmos de incubación, alimentación de pichones, estacionalidad de nidificación y descripciones formales de sus nidos [6]. De todos los eventos de biología reproductiva en las aves (i.e, cortejo, cópula, acarreo de materiales para construcción de nidos, incubación, provisión de alimento para crías, cuidado parental, presencia de individuos con plumaje juvenil, u otros detalles del proceso de anidación), la mayoría de publicaciones en Ecuador se centran en descripciones casuales de nidos (e.g., [7-12]). Pocos son los trabajos que han dado un seguimiento exhaustivo a todo el proceso de nidificación (e.g., [13,14]) o que tienen datos de más de un nido para la misma especie (e.g., [15,16]).

Hay que destacar que artículos y notas cortas sobre historia natural continúan siendo necesarios, y una compilación de estos permitirá una mejor perspectiva de la biología reproductiva y la evolución de las especies [4]. Para tener una efectiva comparación entre datos reproductivos de las especies es necesaria una estandarización de términos y metodologías [17]. El presente trabajo tiene como objetivo estimular a investigadores y observadores de aves a la publicación de datos sobre biología reproductiva y que normalicen la toma de datos en encuentros fortuitos o búsquedas sistematizadas de cualquier tipo de evento de biología reproductiva.

Para este trabajo realicé una revisión general de artículos publicados en revistas indexadas, prioricé mi análisis en artículos más recientes y con énfasis en las aves del Neotrópico. Empleé en el motor de búsqueda Google Académico (https://scholar. google.com), palabras clave como "biología reproductiva", "nidos","aves" y sus respectivas traducciones en inglés. Revisé las metodologías empleadas en los artículos, compilando, junto con mis sugerencias, los datos que deberían ser considerados para registrar un evento reproductivo de la manera más completa posible. Finalmente extraigo los términos habitualmente empleados en los manuscritos, tanto en inglés como en español, y elaboro una definición en base al contexto de los artículos.

La información que se puede obtener en el campo sobre la biología reproductiva de las aves es muy amplia. Detalles puntuales que no requieren más que una atenta observación, pueden proporcionan información valiosa (e.g., primeras descripciones de 
nido para una especie [12, 15], la compilación de eventos reproductivos de una región que da indicios de la temporalidad [7], o el reporte de anidación de especies cuya reproducción se desconocía en el país [10])

Ante el encuentro de cualquier evento reproductivo es importante anotar datos básicos: (i) la identificación de la especie, (ii) la localidad, (iii) la fecha, (iv) la descripción geográfica y ecológica del sitio. En caso de tratarse de un nido se debe incluir una descripción de la estructura en donde este se ubica (e.g. en la copa de un árbol, sobre una estructura construida por humanos, adherido a una planta, etc.). Siempre es útil una fotografía o esquema del nido y fotografías detalladas de la planta a la que está adherida el nido para confirmar la identificación de la misma.

Entre las herramientas importantes para tomar datos de eventos de biología reproductiva están: receptor de sistema de posicionamiento global (conocido por su acrónimo en inglés GPS), cinta métrica (en el campo con una regla pequeña se puede colocar marcar en un palo para medir la altura de un nido que está fuera de alcance, o se puede usar el brazo del investigador para referenciar alturas o profundidades de cavidades), calibrador (para las medidas de nidos huevos y aves, detalladas más adelante), balanza o pesolas, cámara fotográfica (el temporizador de la cámara puede ser muy útil para nidos fuera de alcance, programando al mayor lapso y sujetándola a un palo para llegar al nido) y brújula.

Para las fotografías tomadas en campo es muy útil una cartilla que nos indique la relación de tamaño y de color del objeto fotografiado. Adicionalmente una referencia de la orientación geográfica, que pueda ser posicionada con la brújula, permitirá indicar la dirección de entrada del nido o la ubicación de los huevos. El color puede ser subjetivo al observador o la cantidad de luz del momento, por ello en la cartilla de referencia que sugiero (fig. 1) incluyo la lámina de color de Encycolorpedia (http://encycolorpedia.es/) donde independientemente de la coloración de la imagen impresa o en pantalla, en la fotografía se podrá ubicar la posición en la lámina más similar al objeto fotografiado (e.g., un huevo), posteriormente en http://encycolorpedia.es/ se debe seleccionar con el cursor, la misma ubicación escogida para obtener la descripción del color.

En el trabajo con seres vivos, la ética del investigador es primordial, por ello la toma de datos debe ser muy cuidadosa y consciente, procurando afectar en lo mínimo el desarrollo natural de los eventos, por ejemplo no tratar de rescatar a un juvenil fuera de nido, lo más probable es que esté aprendiendo a volar y sus progenitores estén cerca. La toma de datos de huevos y pichones debe realizarse en ausencia de los progenitores, se sugiere que ante los llamados de alerta, el investigador no debería estar presente por más de 5 minutos [18]. La periodicidad de la toma de datos debe ser considerada por el investigador en función del estrés que se genere en los progenitores. Maclvor et al. [19] encontraron que el monitoreo diario de nidos de Charadrius melodus, no incrementó el riesgo de depredación, sin embargo sugieren que cada visita a los nidos sea desde la mayor distancia y el menor tiempo posibles. Hay evidencias que el disturbio de los investigadores en los nidos no necesariamente involucra efectos negativos en el éxito de reproductivo, incluso algunos predadores se han mostrado más sensibles que las especies estudiadas [20], pero toda precaución es necesaria para minimizar el impacto.
Considerando que las colecciones aún son necesarias, en particular en el Neotrópico [21] y que los huevos y nidos están pobremente representados en los museos nacionales (obs. pers.); es importante realizar colecciones (con los respectivos permisos legales) porque son una gran fuente de información y permiten el desarrollo de diversos estudios [22]. La mayor cantidad de información colectada, registrada con fotografías o descrita en la libreta de campo, permitirá una correcta descripción y comparación con datos previamente publicados de los eventos de biología reproductiva. A continuación detallo algunas variables a considerar:
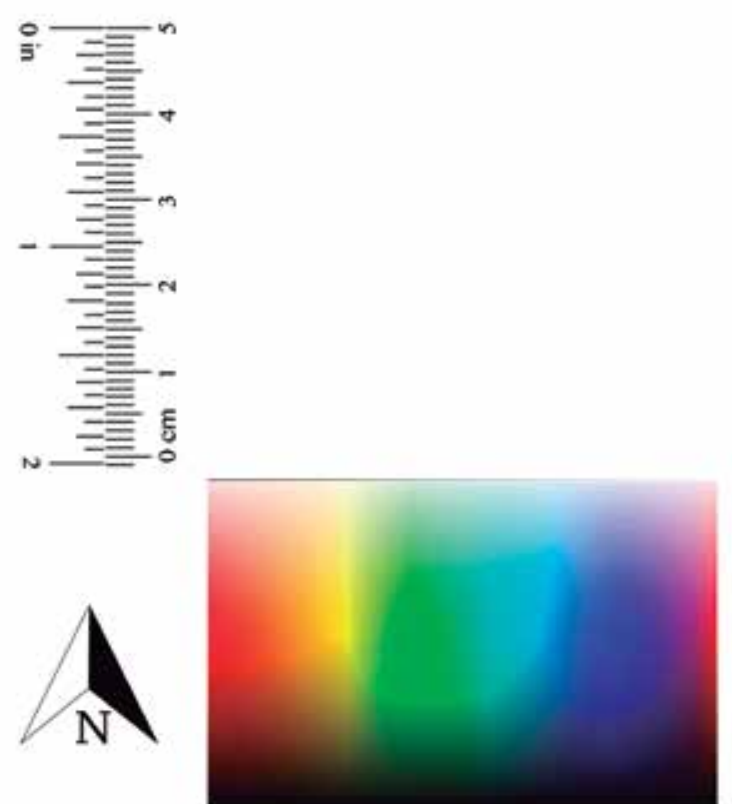

1. Sugerencia de cartilla de referencia para medida, color (Encycolorpedia) y norte magnético, para la toma de fotografías de nidos, huevos o pichones.

\section{FORMA DEL NIDO}

Simon y Pacheco [17] proponen nomenclaturas para varios tipos de nidos, que se agrupan en cuatro principales que se describen a continuación:

1. Simples: sin una estructura notoria, se pueden identificar dos: desnudo (unlined): cuando los nidos están en el suelo, aun si hay una leve excavación o limpieza en el área; y plataforma (platform): cuando algo de material ha sido apilado o vagamente entrelazado, incluso sobre agua.

2. Taza o copa (cup): cuando se asemeja a un recipiente, es importante notar cómo está soportada, si desde la base o lateralmente y a cuántas ramas o plantas está sujeta. También puede ser nombrado como nido abierto (open nest [23]) y ser voluminoso (bulky) o de poca profundidad (shallow/low). 
3. Cerrado (closed): cuando existen paredes que albergan completamente la cámara

1 de incubación; hay que considerar su forma e igualmente desde donde está soportada.

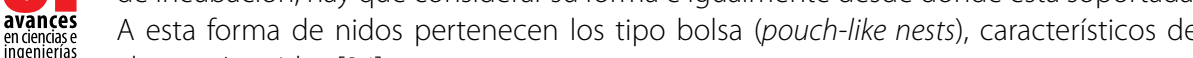
algunos Icteridos [24].

4. Cavidad (cavity): cuando los huevos son depositados dentro de las cavidades naturales o artificiales Por ejemplo es común entre alguna aves como los trogones, usar termitaria / termite nest) para sus nidos [12]. Para este tipo de nidos es importante considerar la presencia o no de un túnel de acceso, su longitud y orientación un espejo bucal y una linterna podrían ser muy útiles para visualizar el contenido.

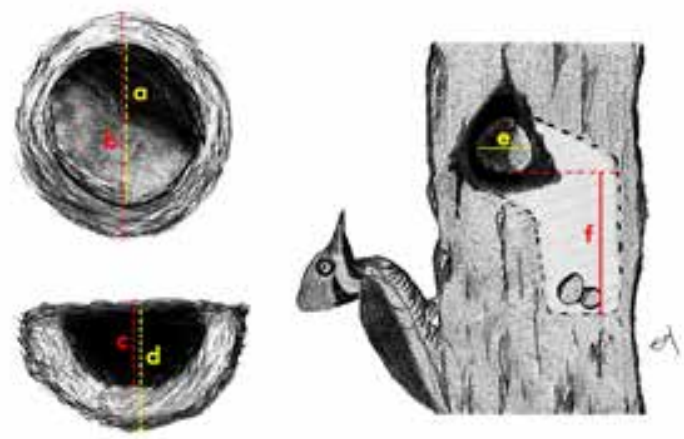

Figura 2. Medidas sugeridas para considerar en un nido: a) diámetro interno, b) diámetro externo, c) profundidad, d) altura, e) diámetro de la entrada, f) profundidad de cavidad (llustraciones: Eliana Montenegro).

\section{ENTORNO DEL NIDO}

Knowlton [25] propone, para un detalle completo del entorno del nido, considerar en un radio de cinco metros a partir del nido y registrar todas las especies vegetales, su diámetro a la altura del pecho (DBH), el tamaño de la copa del árbol donde se encontró el nido, la orientación del nido, su distancia al follaje, distancia al tronco, número de ramas que soportan el nido, el diámetro de estas y el porcentaje de cobertura sobre el nido.

\section{COLOR DEL HUEVO}

El cascaron del huevo de un ave está formado principalmente por carbonato de calcio, de color blanco al ojo humano; la variación del color se da por la interacción de las propiedades físicas y químicas del cascaron y por la presencia de dos pigmentos: biliverdinas (tonalidades azul-verde) y protoporfirinas (colores oxidados, de amarillo a rojo a marrón) [26]. Es importante una clara descripción del color y manchas de los huevos (fig. 4), para esto una fotografía puede ser muy útil y más aún si tiene de fondo una cartilla de referencia de color e. g. (fig. 1).

\section{MEDIDAS}

Nido: dependiendo del tipo de nido se pueden considerar medir diferentes ejes [17], para la mayoría de tipos, aplicaría considerar la distancia desde el suelo al borde inferior de la entrada del nido, su diámetro interno y externo, altura y profundidad. En caso de nidos en cavidades: diámetro de la entrada y profundidad de la cavidad desde el borde inferior de la entrada al piso de la cavidad e. g. [14-16] (fig. 2).

Huevos: largo y ancho mayor; hay que ser muy cuidadosos si se remueven los huevos del nido, tratarlos con precaución y dejarlos en el mismo sitio y posición que fueron tomados. Skutch [23] menciona que en todos sus muestreos midió los huevos sin sacarlos del nido. Incluso el toparlos con el calibrador podría generar fisuras, por lo que es mejor tomar las longitudes dentro del nido y con una cinta o hilo, al cual después se lo pueda medir o fotografiarlos con una referencia de medida y posteriormente establecer sus dimensiones en la computadora.

Aves: se puede tomar varias medidas morfométricas tanto a adultos como a juveniles y polluelos. Winker [27] sugiere considerar: longitud del pico (desde el borde anterior del nostrillo a la punta); longitud de la cuerda de ala; longitud de la cola (insertando una regla con tope en cero, en medio de las rectrices centrales) y longitud tarsometatarso (fig. 3).

\section{Pesos}

Es importante registrar periódicamente los pesos de los huevos, ya que estos van perdiendo peso desde su puesta [8], mientras que su volumen y dimensiones no cambian durante la incubación [28]. También se puede considerar un registro periódico del peso de los pichones [29]. Una vez finalice el proceso de nidificación, puede tomarse el peso seco del nido, también disgregarlo y pesar cada material [11].

\section{Fenología}

Se puede registrar las fechas más significativas: desde la construcción del nido hasta la independencia de las crías para establecer los períodos reproductivos; anotar día y hora de puesta y eclosión de cada huevo, de abandono del nido y días de permanencia con los padres. También se puede registrar el desarrollo del plumaje que tienen los polluelos, realizando una descripción de cómo está el plumaje después de la eclosión, citando en que día empieza a aparecer los cañones y en qué zonas del cuerpo, cuándo las plumas rompen los cañones y describiendo la coloración de las plumas en contraste con el plumaje de adulto [29]; una vez alcanzado el plumaje juvenil se desarrollan diferentes mudas que están vinculadas con aspectos de su ciclo de vida, como la reproducción, el suministro de alimentos o la migración; generándose plumajes nupciales o de verano o reproductivos [30,31].

\section{Ecología}

Varios datos pueden ser muy útiles para visualizar diferencias regionales entre las especies o patrones entre las familias. En este contexto se podría considerar: la

dedicación de cada sexo en la construcción del nido, incubación y acarreo de alimento a pichones [9], especificando la frecuencia de cada actividad (el número de veces) 
y la duración de cada actividad, que se puede expresar como el porcentaje entre el tiempo dedicado a cada actividad sobre el total del tiempo de observación [32]. En e acarreo de alimento se puede considerar los ítems entregados y si estos porcentajes van cambiando con el tiempo, para un detalle más específico de la dieta de la especie. Se puede notar también si los modos en que los adultos entran o salen del nido tienen un patrón específico o no [33], pueden presentar estrategias discretas para evadir depredadores. Para obtener este tipo de información pueden ser muy útiles las cámaras trampa [34]; para especies que no presentan evidente dimorfismo sexual, el sexado molecular y marcaje con anillas resulta óptimo [35].

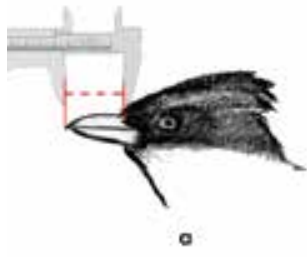

-

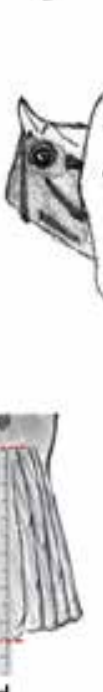

d

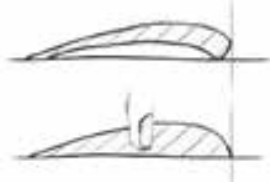

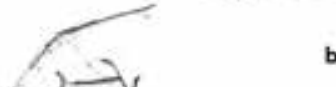

$12(20)$

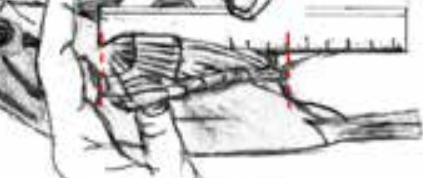

d

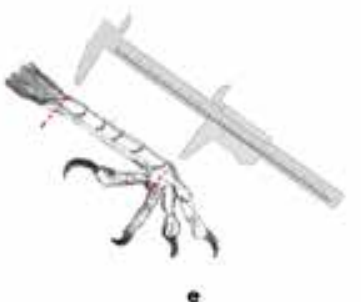

Figura 3. Medidas morfométricas sugeridas a) longitud del pico; b) longitud de la cuerda de ala [superior], longitud del ala plana [inferior], vista frontal; c) Iongitud de la cuerda de ala, vista lateral; d) Iongitud de la cola; e) longitud tarsometatarso (llustraciones: Eliana Montenegro).
Polo fino (sharper end)

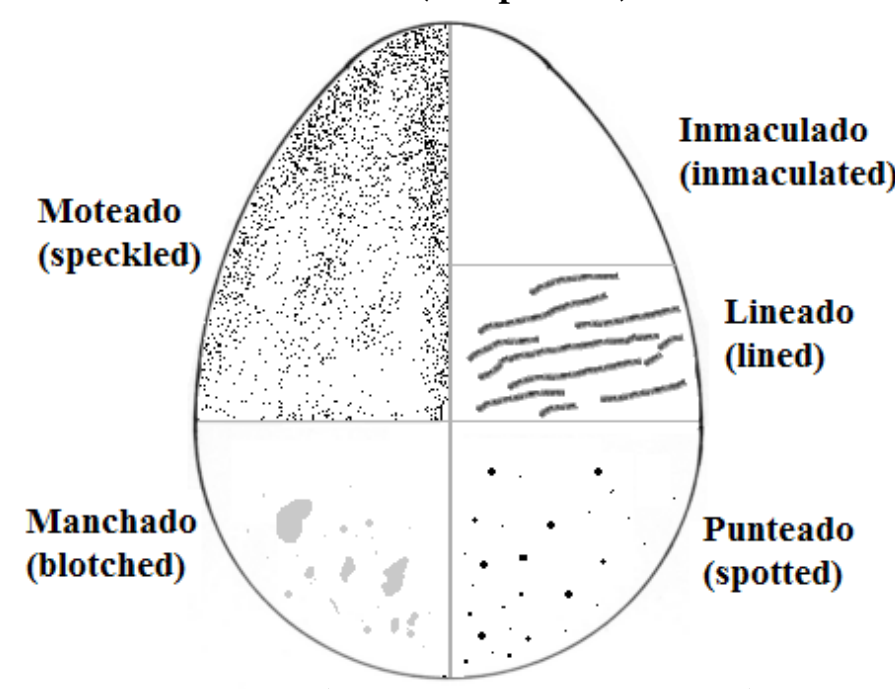

Polo ancho (wider / thicker / blunt end)

Figura 4. Nombres de patrones en huevos con sus traducciones en inglés entre paréntesis

\section{GLOSARIO}

De los trabajos revisados para el presente manuscrito recabo los términos frecuentemente empleados, sugiriendo su correspondiente traducción y definición:

Ahuyentar (flushed): Cuando una persona pasa cerca de un ave y esta se asusta y huye; en ocasiones esto permite evidenciar el nido.

Bolsas fecales (fecal sacs): Son los desechos de los polluelos, que habitualmente son retirados del nido por los progenitores.

Centinela (sentinel): es la actividad de vigilancia de un miembro de la pareja, desde una percha cercana, emitiendo llamados de alerta ante cualquier amenaza para que la pareja escape o se prepare para proteger el nido.

Comisuras (gapes): Uniones en la base del pico que en los pichones usualmente son muy pronunciadas y de colores claros, para que los padres sean más eficientes al alimentarlos.

Descendencia (offspring): el resultado de la reproducción de una pareja de aves van tomando los siguientes nombres de acuerdo a sus clases de edad, en el siguiente orden: pichón, juvenil, inmaduro y subadulto. 
Diente de huevo (egg-tooth): Prominencia calcárea presente en la mayoría de pichones ubicada en la punta del pico para romper la cáscara del huevo al final del periodo de incubación.

Eclosión (hatching): Momento en el que el pichón sale del huevo.

Inmaduro (immature): descendencia de las aves considerada posterior al periodo de dependencia y cuando ha perdido su plumaje juvenil

Juvenil o volantón (juvenil / fledgling): descendencia de las aves considerada desde que fugan del nido, es decir abandonan el nido por sus propios medios, hasta que se independizan del cuidado parental.

Muda (molting): proceso de pérdida natural de plumas y su subsecuente crecimiento de nuevas, por el cual se alcanzan los diferentes plumajes, e. g. plumaje juvenil, plumaje reproductivo.

Nidada (broods): Número de descendientes o aves eclosionadas.

Nidífugo (precocial): cuando el pichón nace con estado de desarrollo avanzado, que le permite movilizarse y auto regular su temperatura; nacen con los ojos abiertos y plumas a lo largo de sus alas y plumón en su cuerpo.

Nidícola (altricial): cuando el pichón salen del huevo sin estar completamente desarrollado, sin plumas y ojos cerrados.

Nido (nest): Estructuras para incubar huevos y criar polluelos, y en algunas especies pueden tener un uso secundario como refugio [36].

Nido comunal (communal nest): cuando varias hembras depositan sus huevos en una misma estructura.

Período de atención al nido (nest attentiveness): Tiempo que el adulto emplea incubando, empollando y alimentando (provisioning) en el nido, no incluye el tiempo que los adultos pasan en las cercanías del nido [32].

Período de construcción del nido (nest-building): desde que el o los adultos empiezan con el acarreo de material hasta que se desova el primer huevo.

Período de dependencia (dependency period): Tiempo desde que eclosiona el primer pichón hasta que toda la descendencia se independizan del cuidado parental.

Período de empollamiento (brooding / nestling): Tiempo desde que los adultos calientan o cubren a los pichones hasta que el último pichón vuela del nido.

Período de incubación (incubation): Tiempo en que los adultos calientan los huevos desde que el último huevo fue puesto hasta que todos hayan eclosionado.
Período de puesta (laying): Tiempo desde que la hembra desova el primer huevo hasta el último.

Pichón (nestling / chick): descendencia de las aves considerada desde que eclosionan os huevos hasta que fugan del nido.

Plumón (down feathers): Plumas de gran superficie pero poca masa lo que les provee suavidad y permite brindar abrigo, constituyen el primer plumaje de los polluelos.

Subadulto (subadult): descendencia de las aves considerada posterior al periodo de dependencia y cuando su plumaje ya es de adulto pero aún no bien definido.

Tamaño de puesta (clutch size): Número de huevos puestos por una hembra en el nido.

Temporadas de cría (breeding seasons): Épocas en las que hay picos de reproducción en las aves; más marcado en las zonas templadas que en los trópicos.

Vainas o cañones (feather sheaths / pin feather / blood feather): La pluma aun envuelta cuando recién emerge de la piel.

Vuelo de amague (flyby): Comportamiento en el que un miembro de la pareja vuela hacia el nido e ingresa, al mismo tiempo que el otro miembro también se dirige al nido, cruzando el sendero del primero y provocando un breve y confuso destello de alas y movimiento, podría ser una estrategia para distraer a los depredadores [37].

La información detallada anteriormente tiene como objetivo que investigadores puedan disponer de elementos para incrementar y optimizar la toma de datos y posterior análisis con la finalidad de estandarizar el conocimiento en esta interesante rama de la ornitología. Finalmente, la conservación de las especies depende de un conocimiento adecuado de su historia natural, especies amenazadas podrían incrementar su población con una adecuada protección de sus áreas de anidación o la implementación de cajas nido, para cualquier alternativa es necesario un conocimiento previo claro.

\section{AGRADECIMIENTOS}

Este manuscrito se vio enriquecido por los comentarios de Carles Barriocanal, Jorge Brito, Tjitte de Vries y revisores anónimos a quienes les agradezco; también por la paciente ayuda con las figuras a Eliana Montenegro. 


\section{aci}

\section{Referencias bibliográficas}

1. Baillie, S.R. (1990). Integrated population monitoring of breeding birds in Britain \& Ireland. Ibis, 132, 151-166. doI http://dx.doi.org/10.1111/j.1474-919X.1990.tb01035.x

2. Xiao, H., Hu, Y., Lang, Z., Fang, B., Guo, W., Zhang, Q., Pan, X. \& Lu, X. (2016), How much do we know about the breeding biology of bird species in the world?. Journal of Avian Biology. doi: http://dx.doi.org/10.1111/jav.00934

3. Marini, M. Â., Borges, F. J., Lopes, L. E., Sousa, N. O., Gressler, D. T., Santos, L. R., Paiva, L. V., Duca, C., Manica, L. T., Rodrigues, S. S., França, L. F., Costa, P. M., França, L. C., Heming, N. M., Silveira, M. B., Pereira, Z. P., Lobo, Y., Medeiros, R. C.S. \& Roper, J.J. (2012). Breeding biology of birds in the Cerrado of central Brazil. Ornitología Neotropical, 23, 385-40.5. Recuperado de https://sora.unm.edu/node/133324

4. Freile, J. F., Greeney, H. F., \& Bonaccorso, E. (2014). Current Neotropical ornithology: Research progress 1996-2011. The Condor, 116(1), 84-96. doi: http://dx.doi.org/10.1650/CONDOR-12-152-R1.1

5. Nilsson, J., Freile, J. F., Ahlman, R., Brinkhuizen, D. M., Greenfield, P. J., \& Solano-Ugalde, A. (2014). Rare birds in Ecuador: Second animal report of the Committee for Ecuadorian Records in Ornithology (CERO). Avances en Ciencias elngenierías, 6: B38-B50. Recuperado de http://Www.usfq.edu.ec/publicaciones/avances/archivo_de_contenidos

6. Greeney, H. F. (2015). Anidación de la avifauna ecuatoriana: dónde estamos y hacia dónde vamos. En D.F. CisnerosHeredia, J.F. Freile, E. Guevara (Eds), Archivos Académicos USFQ, Número 3, Resumenes de la IV Reunion Ecuatoriana de Ornitología (pp. 9). Recuperado de http://www.usfq.edu.ec/publicaciones/archivosacademicos/Documents/ Archivos_Academicos_USFQ_Numero_3.pdf

7. Greeney, H. F., Martin, P. R., Gelis, R. A., Solano-Ugalde, A., Bonier, F., Freeman, B., \& Miller, E. T. (2011). Notes on the breeding of high-Andean birds in northern Ecuador. Bulletin of the British Ornithological Club, 131, 24-31.

8. Greeney, H. F. (2006). The nest, eggs, and nestlings of the Rufous-headed Pygmy-Tyrant (Pseudotriccus ruficeps) in southeastern Ecuador. Ornitologia Neotropical, 17, 589-592. Recuperado de https://sora.unm.edu/node/119796

9. Skutch, A. F. (1947). A nesting of the plumbeous kite in Ecuador. The Condor, (49) 25-31. doi: http://dx.doi. $\operatorname{org} / 10.2307 / 1364425$

10. Pozo-Zamora, G. M., Batallas, D., Echeverría-Vaca, G., \& Garzón, C. (2015). Observaciones sobre el Zambullidor Grande Podiceps major (Aves: Podicipedidae) en Ecuador, con el primer registro de anidación y la descripción de vocalizaciones. Avances en Ciencias e Ingenierrias, 7, B1-B4. Recuperado de http://www.ustq.edu.ec/publicaciones/ avances/archivo_de_contenidos/Documents/volumen_7_numero_1/B1-7-1-2015.pdf

11. Freile, J. F. (2016). Nesting of the Scrub Tanager (Tangara vitriolina) in Andean Ecuador. Ornitologia Neotropical, 26(1), 51-58. Recuperado de http://journals.sfu.ca/ornneo/index.php/ornneo/article/view/12/5

12. Greeney,H. F., \& Cadena-Ortiz, H. (2016) First nest description for Amazonian Trogon Trogon ramonianus, from eastern cuador, and a review of breeding data for Green-backed Trogon T. viridis. Cotinga online 38, 41-42. Recuperado de ttp://www.neotropicalbirdclub.org/wp-content/uploads/2016/02/Greeney-Cadena-Ortiz.pd

13. de Vries, T. (1975). The breeding biology of the Galapagos Hawk, Buteo galapagoensis. Le Gerfaut, 65, 29-57.

14.Greeney, H. F., Kirwan, G. M., \& Miller, E. T. (2012). Nesting biology of the Long-wattled Umbrellabird Cephalopterus penduliger. Part Il: nestling provisioning. Cotinga, 34 online: 23-27. Recuperado de http://www.neotropicalbirdclub. org/wp-content/uploads/2015/03/(C34-Greeney2.pdf

15. Durães, R., Greeney, H., \& Hidalgo, J. R. (2008). First description of the nest and eggs of the Western Striped Manakin (Machaeropterus regulus striolatus), with observations on nesting behavior. Ornitología Neotropical, 19(2), 287-292. Recuperado de https://sora.unm.edu/node/133428

16.Juiña, M. E., Harris, J. B. C., Greeney, H. F., \& Hickman, B. R. (2010). Descripción del nido y cuido parental de la Estrellita Esmeraldeña (Chaetocercus berlepschi) en el occidente del Ecuador. Ornitología Neotropical, 21, 313-322. Recuperado de https://sora.unm.edu/node/133173.

17. Simon, J. E., \& Pacheco, S. (2005). On the standardization of nest descriptions of neotropical birds. Revista Brasilieira de Ornitologia, 13(2), 143-154. Recuperado de http://www4.museu-goeldi.br/revistabrornito/revista/index.php/BJO article/view/2201/pdf_32
18. Martin, T. E., \& Geupel, G. R. (1993). Nest-Monitoring Plots: Methods for Locating Nests and Monitoring Success. Journal of field Ornithology, 64(4), 507-519. Recuperado de https://sora.unm.edu/node/51838

19. Maclvor, L. H. Melvin, S. M., \& Griffin, C. R. (1990). Effects of research activity on piping plover nest predation. The Journal of Wildlife Management, 443-447. doi: http://dx.doi.org/10.2307/380965

20. Ibáñe-Álamo, J. D., Sanllorente, 0., \& Soler, M. (2012). The impact of researcher disturbance on nest predation rates: a meta-analysis. Ibis, 154(1), 5-14. doi: http://dx.doi.org/10.1111/j.1474-919X.2011.01186.X

21. Vuilleumier, F. (1998). The need to collect birds in the Neotropics. Ornitología Neotropical, 9(2), 201-203. Recuperado de https://sora unm.edu/node/119303

22. Green, R. E. \& Scharlemann, J. P. W. 2003. Egg and skin collections as a reference for long-term ecological studies. Bulletin of the British Ornithologists' Club 123: 165-176. Recuperado de http://www.biodiversitylibrary.org/ item/130382\#page/167/mode/1up

23. Skutch, A. F. (1954). Life histories of Central American birds: families Fringillidae, Thraupidae, Icteridae, Parulidae and Coerebidae. Vol. 31. Berkeley: Cooper Ornithological Society.

24. Robinson, S. K. (1985). The Yellow-rumped Cacique and its associated nest pirates. Ornithological Monographs, 36, 898907. doi: http://dx.doi.org/10.2307/40168323

25. Knowlton, J. L. (2010). Breeding records of birds from the Tumbesian region of Ecuador. Ornitologia Neotropical, 21(1), 109-147. Recuperado de https://sora.unm.edu/node/133152

26. Hanley, D., Grim, T., Cassey, P., \& Hauber, M. E. (2015). Not so colourful after all: eggshell pigments constrain avian eggshell colour space. Biology letters, 11(5), 20150087. doi: http://dx.doi.org/10.1098/rsbl.2015.0087

27. Winker, K. (1998). Suggestions for measuring external characters of birds. Ornitología Neotropical, 9, 23-30. Recuperado de https://sora.unm.edu/node/119282

28. Hoyt, D.F. (1979). Practical methods of estimating volume and fresh weight of bird eggs. The Auk, 96(1), 73-77. Recuperado de https://sora.unm.edu/node/23238

29. Greeney, H. F. (2008). Nestling growth and plumage development of the Spotted Barbtail (Premnoplex brunnescens). Kempffiana, 4, 21-29. Recuperado de http://www.museonoelkempff.org/sitio/Informacion/KEMPFFIANA/ Kempffiana\%204(1)/21-29.pdf

30. Howell, S. N., Corben, C., Pyle, P., \& Rogers, D. I. (2003). The first basic problem: a review of molt and plumage homologies. The Condor, 105(4), 635-653. doi: http://dx.doi.org/10.1650/7225

31. Humphrey, P. S., \& Parkes, K. C. (1959). An approach to the study of molts and plumages. The Auk, 76(1), 1-31. doi: http://dx.doi.org/10.2307/4081839. A

32. Wetherell, E., Greeney, H.F., \& Port, J. (2015). Breeding biology of speckled hummingbird Adelomyia melanogenns in eastern Ecuador. Cotinga online, 37, 43-46. Recuperado de http://www.neotropicalbirdclub.org/wp-content/ uploads/2015/03/Wetherell-et-al.pdf

33. Greeney, H. F., Mclean, A., Bücker, A. D., Gelis, R. A., Cabrera, D., \& Sornoza, F. (2006). Nesting biology of the longwattled Umbrellabird (Cephalopterus penduliger). Part I: incubation. Ornitología Neotropical, 17, 395-401. Recuperado de https://sora unm edu/node/119773

34. King, D. I., DeGraat, R. M., Champlin, P. J., \& Champlin, T. B. (2001). A new method for wireless video monitoring of bird nests. Wildlife Society Bulletin, 29(1), 349-353. Recuperado de http://www.jstor.org/stable/3784019

35. Greeney, H. F., Dyrcz, A., Mikusek, R., \& Port, J. (2015). Cooperative breeding at a nest of Slaty-backed NightingaleThrushes (Catharus fuscater). The Wilson Journal of Ornithology, 127(2), 323-325. doi: http://dx.doi.org/10.1676/ wils-127-02-323-325.1

36. Skutch, A.F. (1961). The nest as a dormitory. Ibis, 103(1), 50-70. doi: http://dx.doi.org/10.1111/.j.1474-919X.1961. tb02420.x

37. Wright, Z., Port, J., \& Greeney, H. F. (2017). Male and female parental care in the Golden-rumped Euphonia (Euphonia cyanocephala). Ornitologia Colombiana, 16, eNB07. Recuperado de http://asociacioncolombianadeornitologia.org/ wp-content/uploads/2017/12/1710.pd 